\title{
Web-based Prostate Visualization Tool
}

Cristina Oyarzun Laura, Visual Healthcare Technologies, Fraunhofer IGD and Technical University of Darmstadt, Darmstadt, Germany, cristina.oyarzun@igd.fraunhofer.de

Katrin Hartwig, Visual Healthcare Technologies, Fraunhofer IGD, Darmstadt, Germany

Anna-Sophie Hertlein, Visual Healthcare Technologies, Fraunhofer IGD, Darmstadt, Germany

Florian Jung, Visual Healthcare Technologies, Fraunhofer IGD, Darmstadt, Germany

Jan Burmeister, Visual Healthcare Technologies, Fraunhofer IGD, Darmstadt, Germany

Jörn Kohlhammer, Visual Healthcare Technologies, Fraunhofer IGD, Darmstadt, Germany

Stefan Wesarg, Visual Healthcare Technologies, Fraunhofer IGD, Darmstadt, Germany

Guido Sauter, University Medical Center Hamburg-Eppendorf, Hamburg, Germany

\section{Introduction}

Numerous studies show how important doctor-patient communication as well as the communication between all stakeholders in the clinic are. Doctor-patient communication is usually based on plain text and bar charts. In case of prostate cancer, this complicates the understanding for the patient in an extremely hard moment. Furthermore, the communication between stakeholders is difficult because of the lack of conventions while referring to the location where a biopsy for diagnosis was taken.

\section{Methods}

A web-based communication tool is proposed that incorporates a visualization of the prostate divided into 27 segments according to the PI-RADS protocol to ensure that no ambiguities exist while determining the location of a biopsy punch. The tool allows the pathologists and radiologists to easily insert all relevant findings. The severity of the findings for each punch can be graded as: healthy tissue, prostatic intraepithelial neoplasia, atypical gland tubes or tumour. Furthermore, a tumour is not analysed solely based on its size and location but also using Gleason's grading system, a very relevant metric used for prostate cancer staging. All this information is visualized using a colour code so that the patient can easily understand his current health status.

\section{Results}

The tool has been evaluated by means of a usability study. 7 participants have carried out a series of tasks and filled out a questionnaire based on SUS and USE Questionnaries and the international standard ISO 9241-110. The results highlight the relevance of the tool both for doctor-patient and doctor-doctor communication.

\section{Conclusion}

The proposed tool incorporates all relevant information for the diagnosis of prostate cancer. This information is visualized using a prostate model divided in 27 segments. This tool facilitates the communication between all stakeholders in the clinic and between doctors and patients. The performed user study highlights the suitability of the proposed tool. 


\section{The challenge for coping with informational deficits in palliative care}

Pavel Larionov, Institut für Biomedizinische Technik (IBMT), FB Life Science Engineering (LSE), Technische Hochschule Mittelhessen (THM) - University of Applied Sciences, Gießen, Germany, pavel.larionov@1se.thm.de Tom Jürgens, Institut für Biomedizinische Technik (IBMT), FB Life Science Engineering (LSE), Technische Hochschule Mittelhessen (THM) - University of Applied Sciences, Gießen, Germany, tom.juergens@1se.thm.de; René Happel, Institut für Biomedizinische Technik (IBMT), FB Life Science Engineering (LSE), Technische Hochschule Mittelhessen (THM) - University of Applied Sciences, Gießen, Germany, rene.happel@1se.thm.de; Clara Kilian, Institut für Biomedizinische Technik (IBMT), FB Life Science Engineering (LSE), Technische Hochschule Mittelhessen (THM) - University of Applied Sciences, Gießen, Germany, clara.kilian@lse.thm.de; Pascal Kieser, Institut für Biomedizinische Technik (IBMT), FB Life Science Engineering (LSE), Technische Hochschule Mittelhessen (THM) - University of Applied Sciences, Gießen, Germany, pascal.su-chon.theo.kieser@1se.thm.de; Christian Teichert, Institut für Biomedizinische Technik (IBMT), FB Life Science Engineering (LSE), Technische Hochschule Mittelhessen (THM) - University of Applied Sciences, Gießen, Germany, christian.teichert@1se.thm.de; Wolfgang George, FB Management und Kommunikation (MuK), Technische Hochschule Mittelhessen (THM) - University of Applied Sciences, Gießen, Germany, wolfgang.george@muk.thm.de; Thomas Schanze, Institut für Biomedizinische Technik (IBMT), FB Life Science Engineering (LSE), Technische Hochschule Mittelhessen (THM) - University of Applied Sciences , Gießen, Germany, thomas.schanze@1se.thm.de

\section{Introduction}

As the informational overload hits everyone, the problem of delivering adequate information to the right actor in the right time is crucial. This is of very high importance in the sensitive field of palliative care. We propose a software solution, which will provide an 'allround' support and a guideline for the caregivers and affected persons.

\section{Methods}

It was shown that insufficient integration of caregivers corresponds with an increased risk for not medically indicated relocation or misdirection of patients within and across institutions. After collecting case stories from healthcare specialists, affected relatives and caregivers, the concept of the app was designed with Adobe XD prototyping software and introduced to senior palliative experts, psychologists, pastors, and lawyers for evaluation.

The core functionalities of the app include a specially designed info-hub, a secure data-hub for personal and vital data, lists of essential contacts and important documents, and a smart home hub with suggestions based on the user-specific situations.

\section{Results}

The caregiver app passed the prototyping stage. It is currently being implemented using a cross-platform development environment (Flutter framework). Preliminary results show that guidance and information portioning, provided by the app, increases the awareness and competence of the caregivers. Designed and developed with interoperability in mind, following the standards of the FHIR framework, the app has the potential to be a connecting link to functional platforms for digital health records by giving users and patients control over their own data.

\section{Conclusion}

By making caregivers allies of health care providers, rather than seeing them as a source of instability, it is possible to reduce the number of misdirected palliative patients, i.e. to ensure that they get the intended treatment. By empowering caregivers or affected persons, the app aims to enforce social support, encourage coping skills and to improve the wellbeing of patients and caregivers.

Sponsored by The Federal Joint Committee of Germany. 


\section{Swipe up to smoke less cigarettes! Introducing a mobile Approach-Avoidance Task Application to fight Smoking}

Tanja Joan Eiler, Medical Informatics and Microsystems Engineering, University of Siegen, 57076 Siegen, Germany, tanja.eiler@uni-siegen.de

Benjamin Haßler, Medical Informatics and Microsystems Engineering, University of Siegen, 57076 Siegen, Germany, benjamin.hassler@student.uni-siegen.de

Armin Grünewald, Medical Informatics and Microsystems Engineering, University of Siegen, 57076 Siegen, Germany, armin.gruenewald@uni-siegen.de

Alla Machulska, Department of Clinical Psychology, University of Siegen, 57076 Siegen, Germany, alla.machulska@uni-siegen.de

Tim Klucken, Department of Clinical Psychology, University of Siegen, 57076 Siegen, Germany, tim.klucken@psychologie.uni-siegen.de

Katharina Jahn, Department of Information Systems, University of Siegen, 57076 Siegen, Germany, katharina.jahn@uni-siegen.de

Björn Niehaves, Department of Information Systems, University of Siegen, 57076 Siegen, Germany, bjoern.niehaves@uni-siegen.de

Carl Friedrich Gethmann, Research College FoKoS, 57076 Siegen, Germany, carl.gethmann@ uni-siegen.de

Rainer Brück, Medical Informatics and Microsystems Engineering, University of Siegen, 57076 Siegen, Germany, rainer.brueck@uni-siegen.de

\section{Introduction}

Automatic tendencies to approach addiction-related stimuli have been linked to the development and maintenance of harmful drug use behavior. Recent studies have shown that these automatic approaches can be directly addressed and modified by cognitive bias modification (CBM). However, the training and treatment effects are rather small and compliance could be impaired by time-consuming, multiple laboratory training sessions. In this paper we present a mobile variant of the Approach-Avoidance Task (app-AAT), which is designed to improve the efficiency of CBM training by allowing smokers to access the training at any time with their Android devices, and by offering the option to track consumed cigarettes.

\section{Methods}

Eight people have participated in our feasibility study, four of whom have been assigned to the training group (TG) and four to the control group (CG). After a psycho-education and introduction to the app-AAT, all persons did a home training for two weeks. While in the TG condition $50 \%$ of the stimuli are addiction-related and should be avoided, in the CG $8 \%$ are addiction-related and the interaction is random. Afterwards, the German version of the Mobile App Rating Scale (MARS-G) was used in order to evaluate the quality and usability of the app.

\section{Results}

$75 \%$ of the participants conducted at least one session per day. The results show that the overall rating of the app is 4.2 out of 5 points (engagement: 3.7, functionality: 4.5, aesthetics: 4.4). Only minor changes have to be made before conducting the main study in form of a randomized controlled trial (RCT).

\section{Conclusion}

The app-AAT had a good adherence after the initial training and achieved a high rating by the participants of both conditions. For further evaluation, at least 150 subjects will be recruited for the RCT. In addition, we are currently developing an iOS version of the app-AAT in order to include owners of Apple devices in the study as well. 